\title{
Prevalence and comparisons of alcohol, candy, energy drink, snack, soda, and restaurant brand and product marketing on Twitch, Facebook Gaming and YouTube Gaming
}

\author{
Caitlyn G Edwards ${ }^{1} \odot$, Catherine C Pollack ${ }^{2,3} \odot$, Sara J Pritschet ${ }^{1}$, Keally Haushalter ${ }^{1}$, \\ John W Long' and Travis D Masterson ${ }^{1, *}$ \\ 'Department of Nutritional Sciences, The Pennsylvania State University, State College, PA 16802, USA: ${ }^{2}$ Department \\ of Biomedical Data Science, Geisel School of Medicine at Dartmouth College, Hanover, NH, USA: ${ }^{3}$ Department of \\ Epidemiology, Geisel School of Medicine at Dartmouth College, Hanover, NH, USA
}

Submitted 16 April 2021: Final revision received 20 September 2021: Accepted 18 October 2021: First published online 25 0ctober 2021

\begin{abstract}
Objective: To compare and evaluate the prevalence of food and beverage marketing on the livestreaming platforms Twitch, Facebook Gaming and YouTube Gaming, as well as examine growth of food and beverage marketing on these platforms over a 17-month period of data collection.

Design: Cross-sectional data were analysed across three livestreaming platforms and six food and beverage categories: alcohol, candy, energy drinks, snacks, sodas and restaurants.

Setting: Stream titles of livestreamed events as well as corresponding hours watched on Twitch, Facebook Gaming and YouTube Gaming.

Participants: None.

Results: There were significant differences between the use of food and beverage brand mentions in stream titles across all three studied platforms $(P<0.05)$, as well as hours watched across platforms $(P<0.05)$. Energy drinks dominated food and beverage brand mentions across platforms, followed by restaurants, soda and snacks. All platforms demonstrated growth over the 17-month data collection period. Post-hoc analyses revealed that the COVID-19 pandemic impacted both immediate and sustained growth across all platforms, with the greatest impact observed on the Twitch platform.

Conclusions: Food and beverage marketing as measured through stream titles is widely prevalent across the three most popular livestreaming platforms, particularly for energy drinks. Food marketing on these platforms experienced growth over the past 17 months which was accelerated substantially by the COVID-19 pandemic. Future work should assess the sustained impact this growth may have on marketing practices and eating behaviour.
\end{abstract}

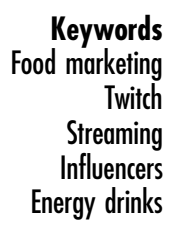

Rates of overweight and obesity continue to rise worldwide $^{(1)}$. The underlying mechanism that contributes to weight gain is the consumption of energy in excess of the body's homoeostatic needs ${ }^{(2)}$. Excess consumption of energy can be driven by a number of factors, one of which includes the endemic presence of marketing for food and beverages in everyday life ${ }^{(3)}$. Food marketing and advertising has long been omnipresent and has been shown to impact both the conscious and unconscious mechanisms that regulate food consumption and choice ${ }^{(4,5)}$. Recent and rapid advancements in technology and shifts in entertainment platforms have contributed to the development of innovative marketing techniques, exacerbating the impacts of the so-called 'obesogenic environment' ${ }^{\text {(6-8) }}$. Therefore, it is vital that the prevalence of food marketing and the techniques employed by food companies on new platforms, such as social media, are monitored to understand the reach and potential impact on food choice and eating behaviour.

'Livestreaming' is a relatively new form of social media entertainment that has seen a dramatic climb in popularity over recent years ${ }^{(9,10)}$. Currently, the three largest 
livestreaming social media platforms in the USA are Twitch (owned by Amazon), YouTube Gaming (owned by Google) and Facebook Gaming (owned by Facebook) ${ }^{(11)}$. All three platforms enable streamers to broadcast live audiovisual content to viewers. In addition to the live broadcast content, streamers are able to directly interact with their audiences in real time. This is accomplished through a live chat feature where viewers of the streaming content can send messages to both the streamer and other viewers, allowing for the community to interact in an organic way. Streamers can also create a profile page which is displayed below their stream where they can provide information to their audiences about their content. This page often includes persistent product advertisements and endorsements ${ }^{(6)}$. Through an advertisement-based revenue model, streamers will often incorporate sponsored products into their entertainment content through a variety of means including overlay ads, product placements, branded mini-games and giveaways. In this way, streamers can take on the role of 'influencers' or individuals with the ability to influence potential buyers of a product or service by promoting or recommending items on social media. This influencer-led advertising content is then presented alongside more traditional website-driven content such as pre- and mid-roll video and banner ads. Advertising on livestreaming platforms therefore combines a number of social media marketing techniques in an overlapping and interactive manner, which is unlike many other social media and entertainment platforms. According to the Reactivity to Embedded Food Cues in Advertising Model, this integration of multiple marketing techniques can influence the advertising effect process ${ }^{(12)}$. The high levels of integration of marketed content on livestreaming platforms may result in less cognitive elaboration and less engagement of consumer defences such as persuasion, knowledge and scepticism, leading to a higher persuasive impact of the marketing strategy ${ }^{(12)}$. While this remains to be thoroughly validated in a livestreaming environment, it has been shown that this integration of passive and participatory techniques has proven to be rewarding, as viewing of livestream events has been shown to be motivated by cognitive, affective, personal integrative, social integrative and tension release motivations ${ }^{(13)}$.

While livestreaming is commonly associated with video gaming, its reach extends far beyond the gaming niche. For example, while the most widely used livestreaming platform Twitch is known for its video game broadcasts, it has expanded its reach to include events such as sports, political rallies and music, among others ${ }^{(14)}$. Since its launch in 2011, Twitch has become the most widely used livestreaming platforms ${ }^{(11)}$. Twitch reports an average viewership of over 2.5 million users at any given moment, 30 million average daily viewers and over 1 trillion min of watched content in $2020^{(15)}$. On a smaller scale, YouTube Gaming reported an average of 871000 viewers streaming at any time during the fourth quarter of 2020 and over 10 billion hours of livestreaming content in $2020^{(16)}$. Facebook Gaming saw a $96 \%$ increase from 2019 in average concurrent viewership to 408000 viewers by the end of 2020, and doubled its hours of streaming content from 0.5 billion at the end of 2019 to over 1 billion by the end of $2020^{(17)}$. This growth has not gone unnoticed by marketing agencies, including those marketing nutrient-poor, energy-dense foods. Previous work identified energy drinks, coffees and teas as the most widely advertised products on Twitch over an 18-month period ${ }^{(6)}$. However, restaurants and food delivery services and sugar-sweetened beverages were most frequently mentioned in chat messages, even though the marketing of these products through streamers was significantly lower than energy drink products. Despite these variations, all products experienced significant growth in exposure over the study period, including sugar-sweetened beverages and candies $^{(6)}$.

Livestreaming services have produced a profound shift in the media landscape, and content creation has shifted from large corporations and organisations to users as content creators, influencers and brand 'affiliates' ${ }^{\prime(18,19)}$. Influencer marketing has proven effective on other internet platforms (e.g. YouTube, Instagram), and marketing of unhealthy snacks to children via influencers in particular has been associated with increased food intake even when the influencer discloses that they are advertising a product ${ }^{(20,21)}$. This marketing is supported by the platforms themselves. As an example, in 2020 Twitch introduced a large number of new influencer-centred marketing techniques to increase revenue on the platform. This included the creation of the 'Twitch Affiliate Program' to help facilitate contact between brands and streamers ${ }^{(22)}$ and the requirement that streamers run a set number of ads per hour of content ${ }^{(23)}$. Alongside these internal changes, as Twitch is a US-based company, the Federal Trade Commission requires all social media influencers, including streamers, to include '\#ad' in the title of a stream when receiving compensation for mentioning a product, regardless of where in the world a streamer may be operating from ${ }^{(24)}$. However, companies have been looking for ways to subvert regulations and partnerships to extend their advertising in unique ways, some more effective than others. For example, a recent Burger King campaign targeted popular streamers and their communities by donating the price of a Burger King meal to a pre-specified group of streamers during one of their streaming sessions. The donation triggered an automated bot to interrupt the stream with a message instructing the streamer and viewers to go to the nearest Burger King and purchase a meal. This type of viral marketing effectively sidestepped \#ad disclosure requirements from the Federal Trade Commission while also implying that the streamer was serving in an influencer capacity for Burger King when no such agreement had been made. This campaign received pushback from many of Twitch's top users and streamers who said such 
marketing ploys were taking advantage of Twitch donation features to avoid paying streamers as formal influencers ${ }^{(25)}$.

Our group has published previously on the marketing techniques and presence of brand exposure for food and beverage items across stream titles, streamer profiles and chat messages on the livestreaming platform Twitch ${ }^{(6)}$. The current study expands upon our previously published work to describe the presence of and potential differences between food and beverage brand mentions across three major livestreaming sites (Twitch, YouTube Gaming and Facebook Gaming). To accomplish this, we examined brand presence across six distinct categories (i.e. alcohol, candy, energy drinks, processed snacks, sodas and restaurants) during a 17 -month time period (i.e. July 2019-November 2020). In addition, we sought to examine the impact the COVID-19 pandemic (i.e. March 2020-November 2020) had on food advertising on these platforms, as it has been reported that advertising budgets shifted significantly to online platforms during this time period $^{(26)}$. This will provide policy makers and researchers an updated understanding on the continued presence and potential growth of food and beverage brand exposure across livestreaming platforms.

\section{Methods}

\section{Generation of brand and product search term}

A list of brands and products was compiled prior to data collection by the research team. The brand list was based on our previously published work ${ }^{(6)}$ but was expanded to include new brands or brands that may have not been advertised on livestreaming platforms previously. To identify new brands, we first consulted updated reports of food marketing trends and practices from the Rudd Center for Food Policy and Obesity ${ }^{(27)}$. We then had two members of the research team examine the profile pages and stream titles of the top 100 worldwide Twitch, YouTube Gaming and Facebook Gaming streamers for current food and beverage brands, both US-based and international, providing sponsorship or being advertised on these platforms ${ }^{(28)}$. Brands that were identified in either the Rudd Center reports or the profile pages and stream titles of the top 100 worldwide streamers were then added to expand our original list of brands. As in our previous work, only core brand names and products were searched, and variants of these brand names were not searched (e.g. 'Coke' was searched while 'Coke Zero' was not, as the base word 'Coke' would still capture this product variant). To account for common misspellings (e.g. 'Gfuel' $v$. "G fuel'), grammatical errors (e.g. 'Reese's $v$. Reeses'), the use of ' $\mathrm{z}$ ' instead of 's' (e.g. 'Cheez-Its' $v$. 'Chees-Its) and general Internet slang (e.g. 'Chick-fil-A' $v$. 'Chikfila'), the finalised list was subjected to an algorithm to generate a variety of spelling variants for each brand ${ }^{(29)}$. In total, 854 search terms were generated (including alternative spellings) to search for 312 brands across six distinct 'pre-identified categories: alcohol, candy, energy drinks (and other caffeinated beverages), processed snacks (foods designed to be eaten outside of meal occasions including: chips, crackers and snack bars), soda (and other sugar-sweetened beverages) and restaurants (and food delivery services that are not restaurant specific). A full list of initial candidate terms is available in the online supplementary material.

\section{Data collection}

All data were extracted from the analytics platform Stream Hatchet $\left(\right.$ (Stream Hatchet SLU) ${ }^{(30)}$. While our previous work was able to cite three metrics of advertising in the livestream environment (i.e. the stream titles of each individual stream, the profile page of each streamer and the chat room logs) limitations have since been placed on publicly available data that can be extracted from the application programming interface by the livestreaming platforms; therefore, it was only possible to access information for stream titles. Additionally, as Twitch was the only platform for which we would have been able to capture chat room data, we chose not to examine it for the purposes of this comparative analysis. Brand prevalence in stream titles and hours of viewership of stream titles were obtained using a Python 3 script that implemented the Selenium library to interact with the Stream Hatchet graphical user interface and automatically and iteratively search each brand and product. Data were collected in December of 2020 for a time period from July 2019 through November 2020. All mentions of food and beverage brands in stream titles were extracted, not just titles containing \#ad, as brand exposure was still a result of use of the food and beverage brand, whether sponsored or not.

\section{Data analysis and post-processing}

All analyses were conducted using the $\mathrm{R}$ language and environment for statistical computing (version 4.0.3, R Studio Team, 2020) using the RStudio Graphical User Interface. All data mining was conducted in the Spyder integrated development environment with Python 3 (version 3.8.0). All code used in this project is openly available at https://osf.io/gm3wd.

To assess brand exposure, we utilised two metrics: stream titles and hours watched. Stream titles refer to the unique mention of a brand or product name in the name or 'title' of the stream. This stream title appears below the livestream content and is visible throughout the streaming experience. The number of unique titles containing each brand or product was quantified and referred to as 'titles' throughout. Hours watched (referred to as 'hours watched' throughout) was calculated as: $h_{\text {total }}=h_{v 1}+h_{v 2}+b_{v n}$, where $h_{\text {total }}$ denotes the total hours of content watched and $b_{v n}$ denotes the hours watched per unique viewer. Therefore, the hours watched is a sum of all 
the time unique viewers spent viewing the livestream content. In addition, because of the a priori knowledge of differences in overall viewership numbers on the three livestreaming platforms of interest ${ }^{(11)}$ (i.e. larger viewership is found on Twitch than the other examined platforms), the ratio of hours watched per title was calculated to standardise potential differences in exposure (i.e. hours watched) to food and beverage products/brands in stream titles across categories and platforms on a monthly basis. The total number of unique titles and total number of hours watched under each title were assessed on a monthly level for each brand in order to examine trends over time. Aggregated monthly totals were collapsed to a total value in order to examine differences between platforms over the study period.

The distribution of titles and hours watched by brand category and across platforms was described using frequencies and differences were examined using $\chi^{2}$ tests. To assess the change in prevalence of brands in both number of unique titles and the number of hours watched over time, linear regression analyses were conducted for each category and for each platform. In these linear analyses, dependent variables were titles or hours, and months (as a discrete variable) were considered independent variables. To evaluate the individual contribution of specific brand titles to the overall brand category, the top five brands in each category were identified for each platform and their percentage contribution to the total stream titles or hours watched was calculated.

In addition to the main analyses, we conducted post-hoc analyses to examine differences in linear growth trends in the data pre- (i.e. July 2019-February 2020) and peri- (i.e. March 2020-November 2020) the COVID-19 pandemic. This was done using interrupted time series analysis with March of 2020 as a breakpoint to analyse the differences. This analysis evaluates the linear trend pre-March, the immediate change in exposure from March to April and the linear trend post-March. Prior to running any statistical tests, a significance level of $\alpha=0.05$ was chosen.

\section{Results}

\section{Analysis of all platforms}

Table 1 displays the top five brands mentioned in stream titles across platforms and categories, as well as the contribution that each brand makes to the total stream titles in each category. There was a significant difference in the number of food and beverage brands from any category mentioned in stream titles $\left(\chi^{2}=900.7, \mathrm{df}=10, P<0.001\right)$ between livestreaming platforms (see Fig. 1). Across all stream titles observed, Twitch was responsible for $90 \%$ of all food and beverage brand mentions in stream titles, while Facebook contributed $6 \%$ and YouTube contributed $4 \%$. Energy drinks were mentioned the most frequently in stream titles across all three platforms ( $74 \%)$, followed by restaurants (9\%), soda ( $8 \%$ ), processed snacks ( $5 \%$ ), alcohol (3\%) and candy (2\%). In terms of hours watched, there was similarly a significant difference in the hours watched under unique titles containing food and beverage brands $\left(\chi^{2}=2068296, \mathrm{df}=10, P<0 \cdot 001\right)$ between livestreaming platforms (see Fig. 1). Twitch was responsible for $90 \%$ of the total number of hours watched, while YouTube Gaming contributed $6 \%$ and Facebook Gaming contributed $4 \%$. The product category with the most hours watched was energy drinks ( $80 \%$ ) followed by restaurants (10\%), soda (3\%), processed snacks (3\%), alcohol (2\%) and candy (2\%).

\section{Twitch}

Food and beverage brands or products were mentioned in 179711 stream titles on the Twitch platform. The predominant category observed in stream titles on Twitch was energy drinks $(73 \%, n 131$ 174) followed by restaurants (9\%, $n 16091)$, soda ( $\%, n 14346)$, processed snacks $(5 \%, n$ 8131), alcohol ( $3 \%, n$ 5180) and candy ( $2 \%$, $n$ 4249). Stream titles containing food and beverage brands or products were viewed for $196645623 \mathrm{~h}$. The predominant contributor to hours watched within Twitch was energy drinks (79\%, n 156181993 h) followed by restaurants (11\% n 20844 136), soda (3\%, n 6682 002), processed snacks (3\%, n 5596166$)$, alcohol (2\%, $n 3443472)$ and candy (2\%, $n 3897$ 854). There was a significant increase in the number of brands mentioned in stream titles in each brand category examined on Twitch during the study period (see Fig. 2): soda (289\% increase; $\beta=7.14,95 \%$ CI 4.82, 9.47, $P<0.001)$, energy drinks (218\% increase; $\beta=67 \cdot 67,95 \%$ CI 54.34, 81.00, $P<0.001)$, restaurants (158\% increase; $\beta=9 \cdot 10,95 \% \mathrm{CI}$ $6 \cdot 81,11.40, P<0.001)$, snacks (148\% increase; $\beta=3.37$, $95 \%$ CI $2 \cdot 19,4.54, P=0.005)$, alcohol (77\% increase; $\beta=1.50,95 \%$ CI $0.97,2.03, P<0.001)$ and candy $(47 \%$ increase; $\beta=1.13,95 \% \mathrm{CI} 0.73,1.53, P<0.001$ ). For hours watched, there was a significant increase in the hours of brand exposure on Twitch during the study period for snacks ( $1081 \%$ increase; $\beta=4364 \cdot 00,95 \%$ CI $2302 \cdot 43$, 6424.98, $P<0.001)$, soda $(719 \%$ increase; $\beta=3712.00$, $95 \%$ CI 2196.08, 5227.32, $P<0.001)$, energy drinks (289\% increase; $\beta=106900, \quad 95 \% \quad$ CI 83 023.03, $130834.90, P<0.001)$ and restaurants $(171 \%$ increase; $\beta=14$ 220.00, 95\% CI 8640.19, 19 797.20, $P<0 \cdot 001)$. No increase was observed for the candy $(P=0.87)$ or alcohol $(P=0.06)$.

\section{Facebook Gaming}

Food and beverage brands or products were mentioned in 11971 stream titles on the Facebook Gaming platform. The predominant category mentioned on stream titles within Facebook Gaming was energy drinks (82\%, $n$ 9807) followed by soda (7\%, $n$ 874), processed snacks 
Table 1 Top five brands in each of the six brand and product categories on Twitch, Facebook live and YouTube Gaming

\begin{tabular}{|c|c|c|c|c|c|c|c|c|c|c|c|c|c|c|c|}
\hline \multirow[b]{2}{*}{ Category } & \multicolumn{5}{|c|}{ Twitch } & \multicolumn{5}{|c|}{ Facebook Gaming } & \multicolumn{5}{|c|}{ YouTube Gaming } \\
\hline & Brands & Titles & $\%$ & Hours & $\%$ & Brands & Titles & $\%$ & Hours & $\%$ & Brands & Titles & $\%$ & Hours & $\%$ \\
\hline \multirow{7}{*}{ Alcohol $(n=56)$} & Total & 5180 & & 3443472 & & Total & 202 & & 188065 & & Total & 186 & & 119243 & \\
\hline & Top 5 & 3591 & 69 & 2878281 & 84 & Top 5 & 160 & 79 & 176768 & 94 & Top 5 & 139 & 75 & 107824 & 90 \\
\hline & White Claw & 1232 & 24 & 288070 & 8 & Modelo & 57 & 28 & 61614 & 33 & Jack Daniels & 49 & 26 & 5239 & 4 \\
\hline & High Noon & 714 & 14 & 364099 & 11 & Heineken & 55 & 27 & 104090 & 55 & Guinness & 43 & 23 & 87076 & 73 \\
\hline & Budweiser & 679 & 13 & 2004709 & 58 & High Noon & 31 & 15 & 8045 & 4 & High Noon & 19 & 10 & 9324 & 8 \\
\hline & Modelo & 555 & 11 & 160828 & 5 & Crown Royal & 9 & 4 & 557 & 0 & Budweiser & 14 & 8 & 4320 & \\
\hline & Heineken & 411 & 8 & 60575 & 2 & White Claw & 8 & 4 & 2462 & 1 & Modelo & 14 & 8 & 1865 & 2 \\
\hline \multirow{7}{*}{ Candy $(n=25)$} & Total & 4249 & & З 897854 & & Total & 117 & & 36761 & & Total & 153 & & 141040 & \\
\hline & Top 5 & 2707 & 64 & 2728254 & 70 & Top 5 & 98 & 84 & 21369 & 58 & Top 5 & 121 & 79 & 66617 & 47 \\
\hline & Oreo & 975 & 23 & 196368 & 5 & Oreo & 56 & 48 & 8708 & 24 & Oreo & 46 & 30 & 36575 & 26 \\
\hline & Snickers & 601 & 14 & 1266912 & 33 & Kit Kat & 22 & 19 & 7060 & 19 & Kit Kat & 28 & 18 & 22823 & 16 \\
\hline & Skittles & 572 & 13 & 178690 & 5 & Skittles & 8 & 7 & 1986 & 5 & Milky Way & 18 & 12 & 3018 & 2 \\
\hline & Reese's & 294 & 7 & 1011653 & 26 & Milky Way & 8 & 7 & 2952 & 8 & Twix & 17 & 11 & 3884 & 3 \\
\hline & Twix & 265 & 6 & 74631 & 2 & Snickers & 4 & 3 & 663 & 2 & Nestle & 12 & 8 & 317 & 0 \\
\hline \multirow[t]{7}{*}{ Energy drinks $(n=28)$} & Total & 131714 & & 156181993 & & Total & 9807 & & 7668962 & & Total & 6299 & & 10691346 & \\
\hline & Top 5 & 129801 & 99 & 155270003 & 99 & Top 5 & 9667 & 99 & 7618159 & 99 & Top 5 & 6114 & 97 & 10609237 & 99 \\
\hline & G Fuel & 77571 & 59 & 117764256 & 75 & Nos Energy & 8628 & 88 & 6319573 & 82 & Nos Energy & 3964 & 63 & 7515311 & 70 \\
\hline & Nos Energy & 40216 & 31 & 18321982 & 12 & Red Bull & 671 & 7 & 495155 & 6 & Red Bull & 1114 & 18 & 1952510 & 18 \\
\hline & Red Bull & 8925 & 7 & 14711888 & 9 & Monster Energy & 145 & 1 & 718294 & 9 & Rockstar Energy & 571 & 9 & 521662 & 5 \\
\hline & Monster Energy & 1921 & 1 & 3951548 & 3 & Starbucks & 127 & 1 & 37772 & 0 & G Fuel & 374 & 6 & 605027 & 6 \\
\hline & Rockstar Energy & 1168 & 1 & 520329 & 0 & G Fuel & 96 & 1 & 47365 & 1 & Monster Energy & 91 & 1 & 14727 & 0 \\
\hline \multirow[t]{7}{*}{ Processed snacks $(n=92)$} & Total & 8131 & & 5596166 & & Total & 628 & & 601400 & & Total & 297 & & 542016 & \\
\hline & Top 5 & 3907 & 48 & 3397483 & 61 & Top 5 & 505 & 80 & 528798 & 88 & Top 5 & 206 & 69 & 521893 & 96 \\
\hline & Doritos & 1252 & 15 & 2193176 & 39 & Dole & 342 & 54 & 245119 & 41 & Goldfish & 89 & 30 & 466191 & 86 \\
\hline & Nutella & 828 & 10 & 167378 & 3 & Kashi & 91 & 14 & 29053 & 5 & Doritos & 50 & 17 & 22036 & 4 \\
\hline & Kraft & 634 & 8 & 305352 & 5 & Belvita & 25 & 4 & 690 & 0 & Nutella & 39 & 13 & 4297 & 1 \\
\hline & Hot Pockets & 601 & 7 & 604092 & 11 & Nutella & 24 & 4 & 19021 & 3 & Pringles & 16 & 5 & 28062 & 5 \\
\hline & Goldfish & 592 & 7 & 127485 & 2 & Doritos & 23 & 4 & 234915 & 39 & Fritos & 12 & 4 & 1307 & 0 \\
\hline \multirow[t]{7}{*}{ Soda $(n=68)$} & Total & 14346 & & 6682002 & & Total & 874 & & 634031 & & Total & 892 & & 292893 & \\
\hline & Top 5 & 9659 & 67 & 4647951 & 70 & Top 5 & 713 & 82 & 498190 & 79 & Top 5 & 710 & 80 & 169962 & 58 \\
\hline & Mountain Dew & 2880 & 20 & 1707029 & 26 & V8 & 303 & 35 & 37681 & 6 & V8 & 373 & 42 & 54493 & 19 \\
\hline & Pepsi & 2249 & 16 & 236494 & 4 & Coke & 148 & 17 & 190100 & 30 & Fanta & 157 & 18 & 15806 & 5 \\
\hline & Coke & 1804 & 13 & 1878258 & 28 & Pepsi & 112 & 13 & 129838 & 20 & Coke & 63 & 7 & 9991 & 3 \\
\hline & Fanta & 1500 & 10 & 555292 & 8 & Fanta & 85 & 10 & 109792 & 17 & Pepsi & 60 & 7 & 38348 & 13 \\
\hline & V8 & 1226 & 9 & 270878 & 4 & Sprite & 65 & 7 & 30779 & 5 & Sprite & 57 & 6 & 51324 & 18 \\
\hline \multirow[t]{7}{*}{ Restaurants $(n=43)$} & Total & 16091 & & 20844136 & & Total & 343 & & 145880 & & Total & 607 & & 676724 & \\
\hline & Top 5 & 11313 & 70 & 9864282 & 47 & Top 5 & 263 & 77 & 106188 & 73 & Top 5 & 450 & 74 & 323161 & 48 \\
\hline & Uber Eats & 3062 & 19 & 3097063 & 15 & KFC & 145 & 42 & 47368 & 32 & KFC & 198 & 33 & 109978 & 16 \\
\hline & $\mathrm{KFC}$ & 2647 & 16 & 918424 & 4 & McDonalds & 42 & 12 & 32020 & 22 & Seamless & 91 & 15 & 21658 & 3 \\
\hline & Chipotle & 2462 & 15 & 3943267 & 19 & Uber Eats & 26 & 8 & 3378 & 2 & McDonalds & 81 & 13 & 148790 & 22 \\
\hline & McDonalds & 1855 & 12 & 1648387 & 8 & Chipotle & 25 & 7 & 21117 & 14 & Uber Eats & 46 & 8 & 19482 & 3 \\
\hline & Taco Bell & 1287 & 8 & 257141 & 1 & Seamless & 25 & 7 & 2305 & 2 & Home Chef & 34 & 6 & 23253 & 3 \\
\hline
\end{tabular}


(a)

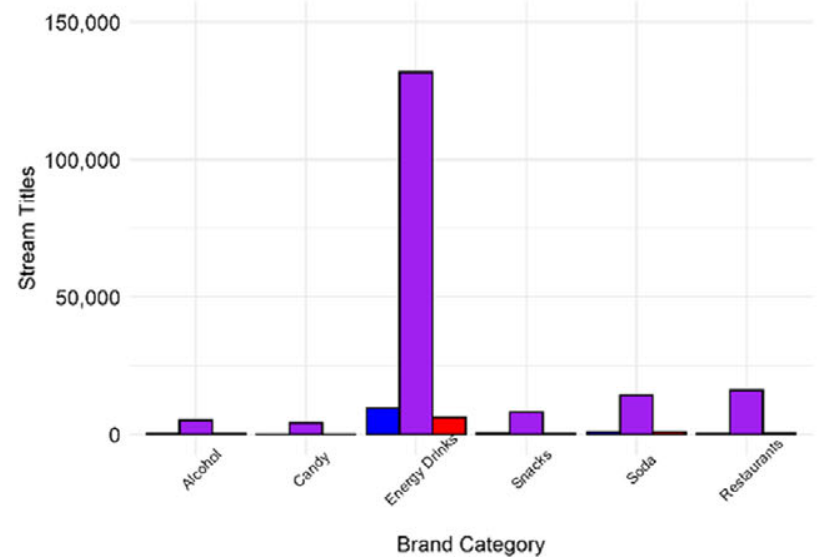

(b)

CG Edwards et al. Hours Watched per Platform per Category

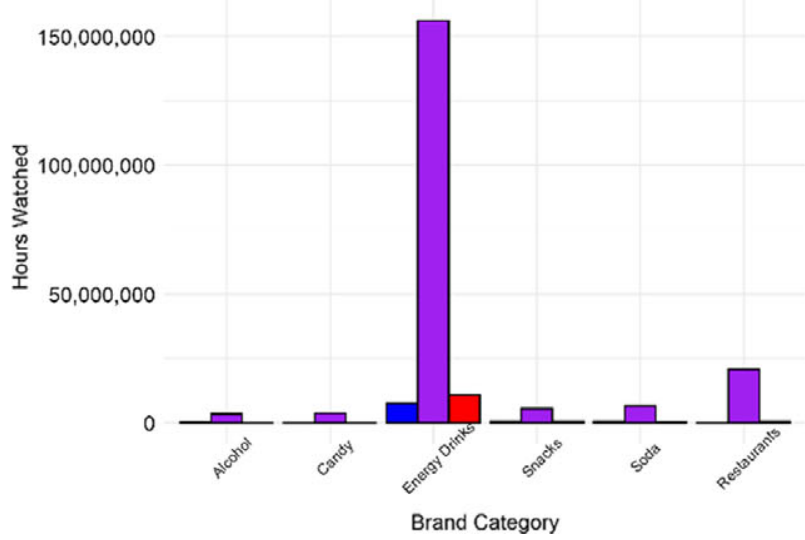

Fig. 1 (colour online) (a) Bar plot depicting the number of stream titles containing a food brand or product name for all platforms (Twitch, Facebook Gaming, YouTube Gaming) across six food or beverage categories (alcohol, candy, energy drinks, snacks, soda,

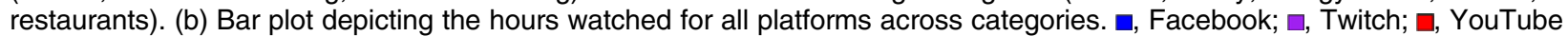

(a)

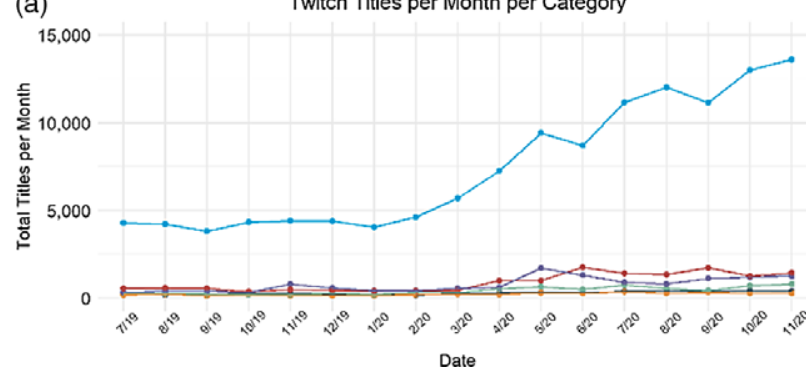

(b)

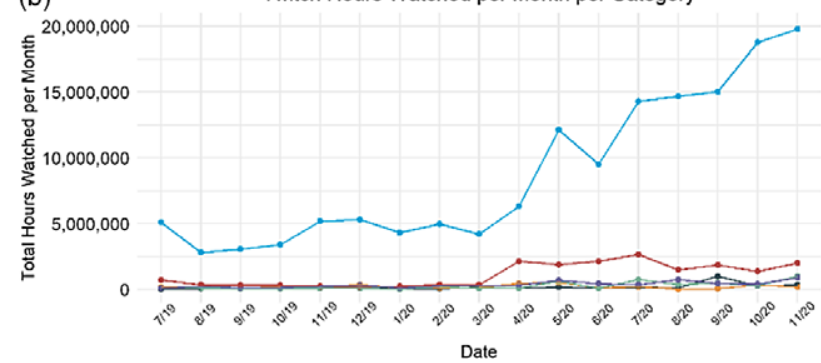

Fig. 2 (colour online) (a) Linear trends of brands mentioned in stream titles across six food and beverage categories on Twitch shown monthly for July 2019-November 2020. (b) Linear trends of hours watched across categories on Twitch shown monthly for July 2019-November 2020. $\rightarrow$, alcohol; $\rightarrow$, candy; $\rightarrow$, energy drinks; $\rightarrow$, restaurants; $\rightarrow$, snacks; $\rightarrow-$, soda
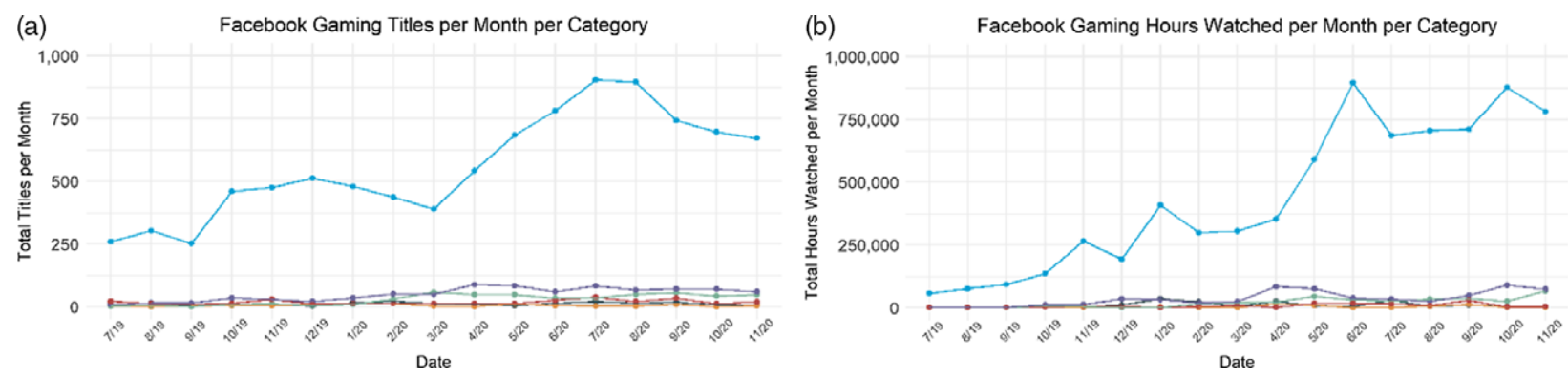

Fig. 3 (colour online) (a) Linear trends of brands mentioned in stream titles across six food and beverage categories on Facebook Gaming shown monthly for July 2019-November 2020. (b) Linear trends of hours watched across categories on Facebook Gaming shown monthly for July 2019-November 2020. $\rightarrow$-, alcohol; $\rightarrow$, candy; $\rightarrow$, energy drinks; $\rightarrow-$, restaurants; $\rightarrow-$, snacks; $\rightarrow-$, soda

(5\%,n 628), restaurants (3\%, $n$ 343), alcohol (2\%, $n$ 202) and candy $(1 \%, n$ 117). Stream titles containing food and beverage brands or products were viewed for $9275099 \mathrm{~h}$. The predominant contributor to hours watched within Facebook Gaming was energy drinks (83\%, $n 7668962$ h) followed by soda $(7 \%, n 634031)$, processed snacks (6\%, n 601 400), restaurants ( $\%, n$ 145 880), alcohol $(2 \%, n 188065)$ and candy $(<1 \%, n 36$ 761). There was a significant increase in the number of brands mentioned in stream titles for (see Fig. 3): snacks (1125\% increase; $\beta=4.88,95 \%$ CI $2 \cdot 58,7 \cdot 17, \quad P=0.003)$, soda $(578 \%$ increase; $\beta=0.37,95 \%$ CI $1.75,0.57, P=0.001)$, energy drinks $(157 \%$ increase; $\beta=3.65,95 \%$ CI $2.60,4.70$, $P<0.001)$ and restaurants (117\% increase; $\beta=0.09,95 \%$ CI $0.003,0 \cdot 17, P=0.04)$. No increase was observed for candy $(P=0.92)$ or alcohol $(P=0 \cdot 30)$. There was a 
Food and beverage livestreaming marketing

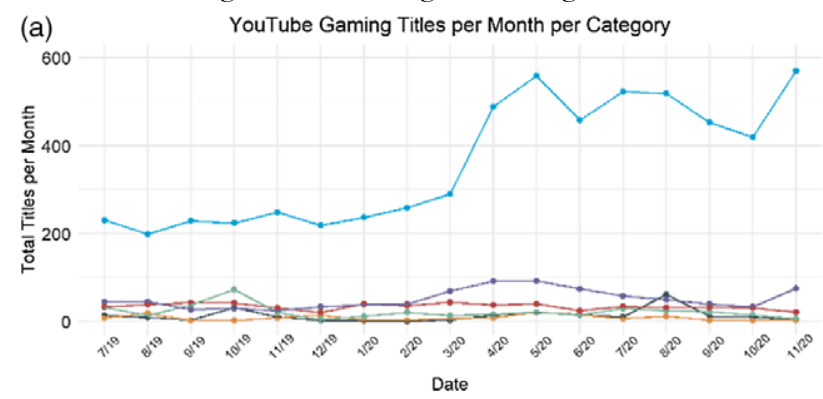

(b)

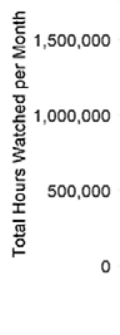

YouTube Gaming Hours Watched per Month per Category

Fig. 4 (colour online) (a) Linear trends of brands mentioned in stream titles across six food and beverage categories on YouTube Gaming shown monthly for July 2019-November 2020. (b) Linear trends of hours watched across categories on YouTube Gaming shown monthly for July 2019-November 2020. $\rightarrow$, alcohol; $\rightarrow$, candy; $\rightarrow$, energy drinks; $\rightarrow$, restaurants; $\rightarrow$, snacks; $\rightarrow-$, soda

statistically significant increase in the hours of brand exposure in soda ( $4072 \%$ increase; $\beta=347 \cdot 00$, $95 \%$ CI 89.71 , 604.26, $P=0.01$ ), snacks (3124\% increase; $\beta=321.40$, $95 \%$ CI 202.86, 439.99, $P<0.001)$, energy drinks (1270\% increase; $\beta=5470,95 \%$ CI $4317.42,6622.62, P<0.001)$ and restaurants (182\% increase; $\beta=101.70,95 \%$ CI $37.73,165.63, P=0.004)$. No increase was observed for candy $(P=0.36)$ or alcohol $(P=0.95)$.

\section{YouTube Gaming}

Food and beverage brands or products were mentioned in 8434 stream titles on the YouTube Gaming platform. The predominant category mentioned in stream titles on YouTube Gaming was energy drinks (75\%, $n$ 6299) followed by soda $(11 \%, n 892)$, restaurants $(7 \%, n 607)$, processed snacks ( $4 \%, n$ 297), alcohol ( $\%, n$ 186) and candy ( $2 \%, n 153)$. Stream titles containing food and beverage brands or products were viewed for $12463262 \mathrm{~h}$. The predominant contributor to hours watched within YouTube Gaming was energy drinks (86\%, n 10691346 h) followed by restaurants $(5 \%, n 676724)$, processed snacks (4\%, n 542 016), soda (2\%, n 292 893), alcohol (1\%, $n 119243)$ and candy (1\%, n 141 040). Energy drinks were the only product category with a significant increase in the number of brands mentioned in stream titles during the study period (see Fig. 4, $246 \%$ increase; $\beta=2.44$, $95 \%$ CI $1.68,3.20, P<0.001$ ). Similarly, only energy drinks experienced a significant increase in the hours of brand exposure on YouTube Gaming during the study window (790\% increase; $\beta=6105.00,95 \%$ CI 2822.98, 9386.52, $P=0 \cdot 001)$.

\section{Adjusted bours per title}

There was a significant difference in the ratio of hours watched per titles across brand category by platform $\left(\chi^{2}=1514.4, \mathrm{df}=10, P<0.001\right.$, Fig. 5). YouTube Gaming had the highest ratio of hours watched per titles $(6528 \mathrm{~h}$ per title), followed by Twitch $(5217 \mathrm{~h}$ per title) and Facebook Gaming ( $4136 \mathrm{~h}$ per title). While Twitch had the highest number of food and beverage brands used in stream titles, as well as the highest hours watched, the only

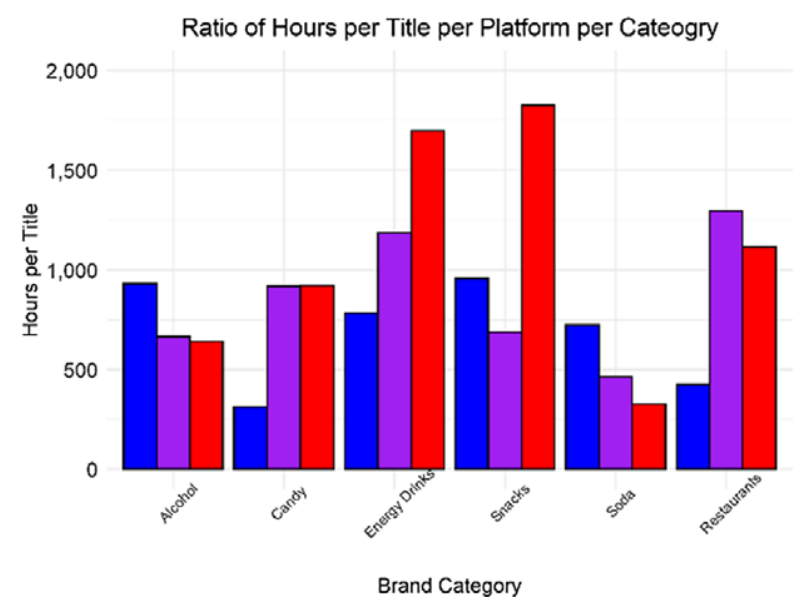

Fig. 5 (colour online) Bar plot depicting the computed ratio of: hours of exposure to a stream title to stream titles containing a food brand or product name for the platforms Twitch, Facebook Gaming and YouTube Gaming across six food and beverage categories. घ, Facebook; $\square$, Twitch; $\square$, YouTube

category in which Twitch had the highest ratio of hours watched per titles was for restaurants. YouTube Gaming had the highest ratio for candy, energy drinks and snack, while Facebook Gaming had the highest ratio for alcohol and soda.

\section{Growth pre- and peri-COVID-19 (March 2020)}

Collapsed across all brand categories, the interrupted time series analysis suggested a significant shift in number of stream titles containing food brand or product names immediately and following March of $2020 \quad\left(\beta_{\text {pre }}=0.88\right.$, $95 \%$ CI $-8.94,10 \cdot 71, P=0.85 ; \beta_{\text {immediate }}=3269,95 \% \mathrm{CI}$ $1341.94,5195.90, P=0.003 ; \beta_{\text {post }}=36.01,95 \%$ CI 23.19 , 48.83, $P<0.001)$, as well as hours watched for the Twitch platform $\left(\beta_{\text {pre }}=5196, \quad 95 \% \quad\right.$ CI -14635.39 , $25028.27, \quad P=0.58 ; \quad \beta_{\text {immediate }}=1913411, \quad 95 \% \quad$ CI $-1976273.90,5803094.94, P=0.31 ; \beta_{\text {post }}=61531,95 \%$ CI 35 658.70, 87 403.68, $P<0.001)$. On Facebook Gaming, stream titles significantly increased prior to March 2020, but did not significantly increase after this date $\left(\beta_{\text {pre }}=1 \cdot 50,95 \%\right.$ CI $0 \cdot 11,2 \cdot 88, P=0 \cdot 04 ; \beta_{\text {immediate }}=63 \cdot 85$, 
$95 \% \mathrm{CI}-207 \cdot 85,335 \cdot 56, P=0.62 ; \beta_{\mathrm{post}}=-0.51,95 \% \mathrm{CI}-$ $2 \cdot 31,1 \cdot 30, P=0.56)$. This phenomenon was also true of hours watched $\left(\beta_{\mathrm{pre}}=1828,95 \%\right.$ CI 623.69, 3032.21, $P=0.006 ; \quad \beta_{\text {immediate }}=50140, \quad 95 \% \quad$ CI $\quad-186053.90$, $286337.22, \quad P=0.65 ; \quad \beta_{\text {post }}=2286, \quad 95 \%$ CI -1342.44 , 1799.70, $P=0.76$ ). On YouTube Gaming, an immediate increase in stream titles was observed in March 2020, but differences between growth before and after this date were NS $\quad\left(\beta_{\text {pre }}=-0.08, \quad 95 \% \quad\right.$ CI $-0.98, \quad 0.83, \quad P=0.86$; $\beta_{\text {immediate }}=263.76, \quad 95 \%$ CI 86.98, 440.54, $P=0.007$; $\left.\beta_{\text {post }}=0.23,95 \% \mathrm{CI}-0.94,1.41, P=0.68\right)$. There were no significant differences in hours watched pre-, peri- or post-March 2020 ( $\beta_{\text {pre }}=197 \cdot 8,95 \%$ CI $-2855 \cdot 81,3251.49$, $P=0.89 ; \quad \beta_{\text {immediate }}=453035 \cdot 1, \quad 95 \% \quad$ CI -145887.98 , $1051958 \cdot 15, P=0 \cdot 13 ; \quad \beta_{\text {post }}=1337,95 \%$ CI $-2646 \cdot 78$, $5320 \cdot 77, P=0 \cdot 48)$.

\section{The impact of COVID-19 on Twitch food}

and beverage brand exposure

Between August 2019 and March 2020, the increase in titles containing energy drink brands on Twitch was NS ( $\left.\beta_{\text {pre }}=1 \cdot 32,95 \% \mathrm{CI}-5 \cdot 58,8 \cdot 21, P=0.69\right)$. The rapid shift to an online environment brought on by COVID-19 introduced an immediate increase in number of titles containing energy drink brands $\left(\beta_{\text {immediate }}=2053 \cdot 23\right.$, $95 \%$ CI $701 \cdot 10$, $8 \cdot 21, P=0.006)$ that was sustained in the following months $\left(\beta_{\text {post }}=29.03,95 \%\right.$ CI 20.03, 3405.37, $P<0.001$, Fig. 6). Similar results were observed for hours watched of energy drinks $\left(\beta_{\text {pre }}=5934,95 \%\right.$ CI $-9014.47,20881 \cdot 90, P=0.41$; $\beta_{\text {immediate }}=298656, \quad 95 \%$ CI $-2633182 \cdot 70, \quad 34736 \cdot 37$, $P=0.829 ; \quad \beta_{\text {post }}=54238,95 \%$ CI $34736.37,73738.99$, $P<0 \cdot 001)$. COVID-19 also impacted stream titles in all other categories: alcohol $\left(\beta_{\text {pre }}=-0.21,95 \%\right.$ CI -0.65 , $0 \cdot 23, P=0.32 ; \beta_{\text {immediate }}=110 \cdot 04,95 \%$ CI 24.02, 196.06, $P=0.02 ; \beta_{\text {post }}=0.73,95 \%$ CI $\left.0.16,1.30, P=0.02\right)$, candy $\left(\beta_{\text {pre }}=-0.12,95 \%\right.$ CI $-0.55,0.32, P=0.56 ; \beta_{\text {immediate }}=$ 95.49, 95\% CI $10 \cdot 12,180 \cdot 86, P=0.03 ; \beta_{\text {post }}=0.34,95 \%$ CI $-0.22,0.91, P=0.21)$, restaurants $\left(\beta_{\text {pre }}=-0.80,95 \%\right.$
CI $-3.48, \quad 1.87, \quad P=0.53 ; \quad \beta_{\text {immediate }}=518.17, \quad 95 \% \quad$ CI $-6.30,1042.64, P=0.05 ; \beta_{\text {post }}=3.94,95 \%$ CI $0.45,7.42$, $P=0.03)$ and processed snacks $\left(\beta_{\text {pre }}=-0.04, \quad 95 \%\right.$ CI $-1.16, \quad 1.08, \quad P=0.93 ; \quad \beta_{\text {immediate }}=197.76, \quad 95 \%$ CI $-21.79,417 \cdot 30, P=0.07 ; \beta_{\text {post }}=1 \cdot 10,95 \%$ CI -0.36 , $2 \cdot 60, P=0 \cdot 13)$. COVID-19 impacted hours watched for restaurants $\left(\beta_{\text {pre }}=-1402,95 \% \mathrm{CI}-6882 \cdot 15,4078 \cdot 69, P=0 \cdot 59\right.$; $\beta_{\text {immediate }}=1382893,95 \%$ CI 307 999.85, 2457787.04, $P=0.02 ; \quad \beta_{\text {post }}=3328, \quad 95 \% \quad$ CI $-3821.40, \quad 10478.05$, $P=0.33$ ), but did not have an impact on the hours watched for alcohol, candy or processed snacks, or soda (all $P^{\prime} \mathrm{s}>0 \cdot 05$ ).

\section{The impact of COVID-19 on Facebook Gaming food and beverage brand exposure}

Prior to March 2020, the use of energy drink brand names in stream titles did not significantly increase $\left(\beta_{\text {pre }}=1 \cdot 13,95 \%\right.$ CI $-0.17,2 \cdot 43, P=0.05)$. COVID-19 had an immediate effect on energy drink brand names in stream titles $\left(\beta_{\text {immediate }}=27 \cdot 12,95 \%\right.$ CI $\left.-227 \cdot 04,282 \cdot 10, P=0 \cdot 005\right)$, but the following growth was not statistically significant $\left(\beta_{\text {post }}=0.10, \quad 95 \%\right.$ CI $\left.-1.83,1.55, \quad P=0.08\right)$. Hours watched of energy drinks were significantly increasing prior to March 2020 but not after $\left(\beta_{\text {pre }}=1473,95 \%\right.$ CI $258.14, \quad 2687.94, \quad P=0.02 ; \quad \beta_{\text {immediate }}=29380, \quad 95 \%$ CI $-208897 \cdot 08,267666 \cdot 81, P=0.79 ; \beta_{\text {post }}=449 \cdot 3,95 \%$ CI $-1135 \cdot 65,2037 \cdot 24, P=0.55)$. COVID-19 also impacted stream titles in other categories: alcohol $\left(\beta_{\text {pre }}=0.08,95 \%\right.$ CI $0 \cdot 02,0 \cdot 14, P=0 \cdot 01 ; \beta_{\text {immediate }}=-7 \cdot 12,95 \% \mathrm{CI}-18.65$, $4.41, \quad P=0.21 ; \quad \beta_{\text {post }}=-0.08, \quad 95 \% \quad$ CI $-0.16, \quad 0.006$, $P=0.04)$, candy $\left(\beta_{\text {pre }}=0.06,95 \%\right.$ CI 0.02, 0.09, $P=0.003$; $\beta_{\text {immediate }}=-9.56,95 \% \mathrm{CI}-16.45,2.66, P=0.01 ; \beta_{\text {post }}=$ $-0 \cdot 06,95 \%$ CI $-0 \cdot 10,-0.01 P=0.01)$, processed snacks $\left(\beta_{\text {pre }}=0.09, \quad 95 \% \quad\right.$ CI $\quad-0.0006, \quad 0.17, \quad P=0.05$; $\beta_{\text {immediate }}=2.71,95 \%$ CI $9.98,44.26, P=0.005 ; \beta_{\text {post }}=$ $-1 \cdot 01, \quad 95 \%$ CI $-0.22, \quad 0 \cdot 13, \quad P=0.08)$ and soda $\left(\beta_{\text {pre }}=0.16,95 \%\right.$ CI $0.03,0.29, \quad P=0.02 ; \quad \beta_{\text {immediate }}=$ $23 \cdot 62,95 \%$ CI $-1 \cdot 04,48 \cdot 29, P=0 \cdot 06 ; \beta_{\text {post }}=-0 \cdot 18,95 \%$
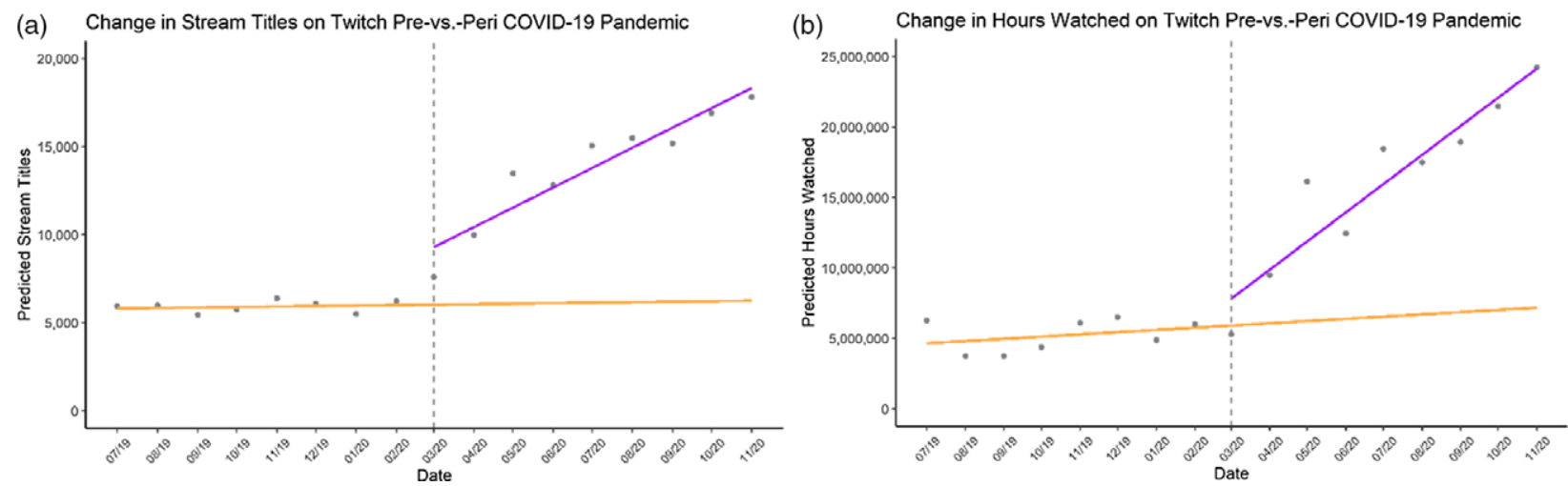

Fig. 6 (colour online) (a) Growth trends for the number of food and beverage brand names used in stream titles across six food and beverage categories on the Twitch Platform pre-March 2020 (yellow line) $v$. after March 2020 (purple line). (b) Growth trends for hours watched on Twitch pre-March 2020 (yellow line) v. after March 2020 (purple line). Note that the yellow line post-March 2020 is a projection of the trend had the COVID-19 pandemic not occurred and is not reflective of the true values 
CI $-1.04,48.29, P=0.04)$. COVID-19 did not have an impact on the use of restaurant names in stream titles $(P>0.05)$. COVID-19 did not have an impact on the hours watched for alcohol, candy, processed snacks, restaurants or soda (all $P>0.05$ ).

\section{The impact of COVID-19 on YouTube Gaming food} and beverage brand exposure

COVID-19 had an immediate effect on the presence of energy drink $\left(\beta_{\text {pre }}=0.15,95 \%\right.$ CI $-0.53,0.83$, $P=0.64 ; \quad \beta_{\text {immediate }}=173.84, \quad 95 \%$ CI $40.79, \quad 306.90$, $\left.P=0.02 ; \beta_{\text {post }}=0.26,95 \% \mathrm{CI}-0.62,1.15, P=0.53\right)$ and soda $\quad\left(\beta_{\text {pre }}=-0.02, \quad 95 \% \quad\right.$ CI $-0.18, \quad 0.13, \quad P=0.76$; $\beta_{\text {immediate }}=51.33, \quad 95 \%$ CI $20.57, \quad 82.09, \quad P=0.003$; $\left.\beta_{\text {post }}=-0 \cdot 13,95 \% \mathrm{CI}-0 \cdot 34,0 \cdot 07, P=0 \cdot 19\right)$ brand names in stream titles. COVID-19 did not have an impact on the use of alcohol, candy, restaurants or processed snack brands mentioned in stream titles (all $P^{\prime} \mathrm{S}>0.05$ ). COVID-19 did not have an impact on the hours watched for any brand category on YouTube Gaming (all $P^{\prime} \mathrm{S}>0.05$ ).

\section{Discussion}

This study expands our previously published work on the platform Twitch and describes the presence of, and potential differences between, food and beverage brand exposure on three popular livestreaming sites (Twitch, Facebook Gaming and YouTube Gaming) across six distinct categories (alcohol, candy, energy drinks, processed snacks, soda and restaurants) during a time period of 17 months (July 2019-November 2020). Of these platforms, Twitch was responsible for generating the greatest number of stream titles containing mentions of food and beverage brands and products. Additionally, Twitch was responsible for the majority of hours viewed under these food and beverage brands. This is not surprising given that Twitch is currently the largest livestreaming platform and has structured programmes to drive advertising on their platforms ${ }^{(11,22)}$. Recent work highlights that self-reported exposure to digital advertising for restaurants and food delivery services has been associated with an increased risk of developing obesity ${ }^{(32)}$. Therefore, characterising the prevalence and growth of food and beverage marketing on livestreaming platforms is an important step in understanding the link between food and beverage marketing and rising rates of overweight and obesity.

While the scale of growth between platforms was different in absolute terms, increases were observed in food brand exposure across all platforms. Although we note that growth on Facebook Gaming and YouTube Gaming was found most notably among energy drinks, increases were also observed for restaurants and sodas. These findings extend our previous work and demonstrate that the livestreaming environment, regardless of platform, is heavily comprised of food and beverage marketing and continuing to grow. Across platforms, energy drinks were the most commonly marketed food and beverage product. Energy drinks contain not only high levels of caffeine but also sugar, ingredients with stimulating properties and understudied novel ingredients ${ }^{(33,34)}$. Energy drinks are packaged in 8-32 oz containers and may contain between 80 and 140 mg of caffeine per $8 \mathrm{oz}$ serving, making it very easy for an adult to reach the upper limit recommendation of daily caffeine intake of $400 \mathrm{mg} / \mathrm{d}^{(35)}$. Important to note is that for children and adolescents, an upper limit of $100 \mathrm{mg} / \mathrm{d}$ has been proposed, which is met with one serving of many popular energy drinks ${ }^{(35,36)}$. Recent work has also linked video game usage to greater energy drink intake ${ }^{(37)}$, and links have been made between the high caffeine levels in energy drinks and self-reported negative physiological health effects among adolescents and adults, including caffeine toxicity, caffeine dependence and caffeine withdrawal ${ }^{(37,38)}$. The dominance of energy drinks was driven by two brands in particular: G FUEL (which accounted for $59 \%$ of all energy drink titles on Twitch) and NOS Energy (which accounted for $88 \%$ of all energy drink titles on Facebook Gaming and $63 \%$ of all energy drink titles on YouTube Gaming, respectively). While these numbers may represent a high global presence of energy drink marketing, they may also be specific to targeted ad campaigns and sponsored streams. For example, G FUEL, the self-proclaimed 'official energy drink of esports', ran one of the most successful ad campaigns on the Twitch platform in 2020, drawing in over 524000 h of viewership by teaming up with a single top streamer ${ }^{(11,39)}$. To better understand which livestreaming platform provided the greatest exposure per title, we examined a ratio of hours watched per number of stream titles on a given platform. This gives some specific insight into the potential impact of a single stream title on a specific platform despite disparities between viewer counts across platforms. For example, Twitch has substantially more users than the other platforms, but it also has more streamers. As a result, this could dilute the potential impact of a single sponsored stream on the platform. Even though Facebook Gaming and YouTube Gaming have fewer overall viewers, our data suggest that sponsored streams on these platforms may still have a significant impact on generating exposure for brands when key streamers are utilised to promote said brand. Future work is needed to fully characterise the potential differences between demographic characteristics of viewers across platforms to fully understand the differences in marketing on different livestreaming platforms.

Our results also demonstrate that COVID-19 had a significant immediate and sustained impact on the presence of brand exposure on livestreaming platforms. This is in line with reports from marketing research that indicates a large shift of marketing dollars to online platforms in response to COVID-19(26). Indeed, there seems to be some agreement that advertising on digital platforms 
(including novel technologies such as livestreaming) is likely to continue to grow at a faster rate than previously expected $^{(26)}$. Time spent online had already been labelled 'unprecedented', but shelter-in-place orders and the transition to online-based work and school pushed hours spent online to record peaks ${ }^{(40)}$. Livestreaming has traditionally been considered a niche market for video gamers, yet rapid technological shifts have facilitated the movement of daily events to livestreaming: children have begun livestreaming school and social events like virtual birthday parties ${ }^{(40)}$, political figures have livestreamed video games as well as historic events ${ }^{(41)}$ and reality TV, sports and competition shows began trending across livestreaming platforms due to restrictions placed on in-person filming ${ }^{(42)}$. Livestreaming is becoming increasingly commonplace, and according to these findings as well as our previous work, food and beverage marketing may continue to grow as well ${ }^{(6)}$.

While we were able to highlight the massive amounts of brand exposure and corresponding rapid growth on livestreaming platforms, future work is needed to understand the sustained effect this will have on both future marketing strategies and eating behaviours. The role of regulation of marketing on these platforms has been of debate, particularly as it pertains to more vulnerable populations such as children. Worldwide regulations towards food marketing practices vary greatly. For example, in the United Kingdom, government has taken steps towards limiting food and beverage exposure towards children, while any regulation in the USA is essentially non-existent ${ }^{(43)}$. Regulations towards marketing unfortunately lag far behind new forms of technology and media, and those that do exist are aimed towards television advertisements, despite research that suggests that this is becoming a decreasingly relevant form of marketing ${ }^{(44)}$. While data surrounding viewership demographics of Facebook Gaming and YouTube Gaming are limited, nearly half of all Twitch accounts belong to users between the ages of $18-34$, and $21 \%$ belong to individuals aged $13-17$ years ${ }^{(45)}$. However, when creating an account users self-report their age with no verification, so it is possible that a large percentage of viewers is younger than these reported statistics. Additionally, livestreaming on many 'mature' platforms can be viewed without needing an active account by simply passing a self-reported age-check question. Even when disregarding these issues, the high prevalence of young viewership is concerning, as evidence suggests that children are not fully aware of the persuasive intent of marketing and tend to accept advertising as truthful, accurate and unbiased ${ }^{(46,47)}$. The evidence base on the impact of advertising exposure among youth is large and growing, with strong findings linking exposure to changes in consumption $^{(48-50)}$. Additionally, this work is the first to highlight the large prevalence of brand exposure for alcohol on livestreaming platforms. Evidence suggests that alcohol advertising and promotion may increase the likelihood that adolescents will start to use alcohol, and that those who begin drinking as an adolescent are more likely to experience alcohol dependence later in life ${ }^{(51,52)}$. Therefore, present work highlights a need to include new forms of media and marketing (including livestreaming) in future conversations regarding regulation of food, beverage and alcohol marketing and to more fully examine the effects of marketing on adolescents, which has been a traditionally understudied group in the food marketing literature ${ }^{(53)}$.

This study drew its strengths from its novel comparison of brand exposure across multiple popular livestream platforms, yet it is not without limitations. First, we were only able to analyse hours watched as they related to stream titles, while previous work allowed us a more comprehensive view of the advertising on the entire platform (such as streamer profiles). While stream titles and hours watched are able to capture a broad view of brand exposure, livestreaming platforms are abundant with other types of simultaneous brand exposure such as video ads, banner ads, overlay ads, product placements and promotional giveaways, to name a few. Therefore, companies have the ability to simultaneously advertise in a number of different ways on livestreaming platforms, which then may spread onto other social media accounts managed by the streamer and their team. These multiple types of advertising and their potential widespread reach may work synergistically to increase brand recall, positive brand attitude and actual purchasing or food choice behaviour, and future work is needed to fully explore these potential synergistic relationships. Therefore, the results presented here are likely a conservative estimate of the true amount of brand exposure on these platforms as we only accounted for the use of food and beverage brands or products in stream titles, and did not account for other potential accompanying marketing tactics. Second, our analysis was not restricted to including the disclosure of an advertising campaign through the use of hashtag (\#) indicators such as '\#ad' or '\#sponsored'. This was a conscious choice, as limiting the search to hashtag indicators would have caused us to miss naturally occurring brand exposure and potential overlap with traditional marketing campaigns (e.g. users livestreaming themselves playing branded mini-games even though the streamer is not sponsored by the brand). Further, our main interest was to comprehensively measure the amount of exposure of food and beverage brands, which takes place regardless of the presence of \#ad. Finally, the brands of interest were developed from a published list of brands that advertised on Twitch and was updated by looking at the profiles and streams of the top 100 streamers on Twitch, Facebook Gaming and YouTube Gaming. As a result, brands that were not on the list or began advertising after our search period could have been missed. However, given the extensive list of brands included in the search, the number of brands missed is likely minimal. 
This work highlights the continued high levels of exposure to food and beverage brands and products on the livestreaming platforms Twitch, Facebook Gaming and YouTube Gaming, and found that most of the brand exposure was derived from energy drink marketing. It is also the first study to model the real-world impact of the COVID-19 pandemic on food and beverage brand marketing growth on livestreaming platforms. These findings indicate that food and beverage marketing on livestreaming platforms is an important component of online marketing practices and should continue to be monitored.

\section{Acknowledgements}

Acknowledgements: None. Financial support: None. Conflict of interest: There are no conflicts of interest. Authorship: T.D.M. and C.C.P. designed the study. C.G.E. performed data collection, statistical analyses and wrote the first draft of the paper. All authors (C.G.E, C.C.P., S.J.P, K.H., J.W.L, T.D.M.) provided valuable feedback of final submission. Ethics of human subject participation: $\mathrm{n} / \mathrm{a}$.

\section{Supplementary material}

For supplementary material accompanying this paper visit https://doi.org/10.1017/S1368980021004420

\section{References}

1. Fryar CD, Carroll MD \& Afful J (2020) Prevalence of Overweight, Obesity, and Severe Obesity Among Adults Aged 20 and Over: United States, 1960-1962 through 2017-2018. NCHS Health E-Stats 2020. [Internet]. https:// www.cdc.gov/nchs/data/hestat/obesity-adult-17-18/obesityadult.htm (accessed March 2021).

2. Liu CM \& Kanoski SE (2018) Homeostatic and non-homeostatic controls of feeding behavior: distinct $v$. common neural systems. Physiol Behav 193, 223-231.

3. Harris JL, Pomeranz JL, Lobstein T et al. (2009) A crisis in the marketplace: how food marketing contributes to childhood obesity and what can be done. Annu Rev Public Health 30, 211-225.

4. Zimmerman FJ (2011) Using marketing muscle to sell fat: the rise of obesity in the modern economy. Annu Rev Public Health 32, 285-306.

5. Cohen DA \& Farley TA (2008) Eating as an automatic behavior. Prev Chronic Dis 5, 1-7.

6. Pollack CC, Kim J, Emond JA et al. (2020) Prevalence and strategies of energy drink, soda, processed snack, candy and restaurant product marketing on the online streaming platform Twitch. Public Health Nutr 23, 2793-2803.

7. Wood B, Williams O, Nagarajan V et al. (2021) Market strategies used by processed food manufacturers to increase and consolidate their power: a systematic review and document analysis. Globalization Health 17, 1-23.
8. Boyland E, Thivel D, Mazur A et al. (2020) digital food marketing to young people: a substantial public health challenge. Ann Nutr Metab 76, 6-9.

9. Anderson M \& Jiang J (2018) Teens, Social Media \& Technology 2018. Pew Research Centre Internet and Technology. https://www.pewresearch.org/internet/2018/ 05/31/teens-social-media-technology-2018/ (accessed November 2021).

10. Bründl S \& Hess T (2016) WHY do users Broadcast? Examining Individual Motives and Social Capital on Social Live Streaming Platforms. Pacific Asia Conference on Information Systems (PACIS 2016). https://aisel.aisnet.org/ pacis2016/332/ (accessed November 2021).

11. Hatchet S (2020) Video Game Streaming Trends Report: 2020 Yearly Report. https://insights.streamhatchet.com/streamhatchet-2020-yearly-report-1 (accessed November 2021).

12. Folkvord F, Anschütz DJ, Boyland E et al. (2016) Food advertising and eating behavior in children. Curr Opin Behav Sci $\mathbf{9}$, 26-31.

13. Sjöblom M, Törhönen M, Hamari J et al. (2017) Content structure is king: an empirical study on gratifications, game genres and content type on Twitch. Comput Hum Behav 73, 161-171.

14. Bowman J (2020) The Best Non-Gaming Twitch Channels, According to Twitch Streamers. https://nymag.com/ strategist/article/best-non-gaming-twitch-channels.html (accessed November 2021).

15. Twitch (2021) Facts and Figures. https://www.twitch.tv/p/ press-center/ (accessed September 2021).

16. Clement J (2021) Average Number of Viewers of YouTube Gaming Live 2020. STATISTA. https://www.statista.com/ statistics/761100/average-number-streamers-on-youtubegaming-live-and-twitch/\#: :text (accessed November 2021).

17. May E (2020) Streamlabs and Stream Hatchet Q4 Live Streaming Industry Report. https://blog.streamlabs.com/ streamlabs-and-stream-hatchet-q4-live-streaming-industryreport-a898c98e73f1\#: : text (accessed November 2021).

18. Cha M, Kwak H, Rodriguez P et al. (2007) I tube, You Tube, Everybody Tubes: Analyzing the World's Largest User Generated Content Video System. Proceedings of the ACM SIGCOMM Conference on Internet Measurement IMC. https://dl.acm.org/doi/abs/10.1145/1298306.1298309 (accessed November 2021).

19. Hilvert-Bruce Z, Neill JT, Sjöblom M et al. (2018) Social motivations of live-streaming viewer engagement on Twitch. Comput Hum Behav 84, 58-67.

20. Coates AE, Hardman CA, Halford JCG et al. (2019) Social media influencer marketing and children's food intake: a randomized trial. Pediatrics 143, e20182554.

21. Coates AE, Hardman CA, Halford JCG et al. (2019) The effect of influencer marketing of food and a "protective" advertising disclosure on children's food intake. Pediatr Obes 14, 1-9.

22. Mikkimarvel (2020) Everything You Need to Know About 'Affiliate' on Twitch. Streamer Sq. https://streamersquare. com/everything-you-need-to-know-about-affiliate-on-twitch/ (accessed November 2021).

23. Stephen B (2020) Twitch is Testing Mid-Roll Ads that Streamers can't Control. The Verge2. https://www. theverge.com/2020/9/15/21437787/twitch-midroll-ad-affiliatepartner-cpm (accessed November 2021).

24. Federal Trade Commission (2009) Guides Concerning the Use of Endorsements and Testimonials in Advertising. Washington, DC: Federal Trade Commission.

25. Chalk W (2020) Twitch: the Streamers Furious at Burger King. BBC News. https://www.bbc.com/news/newsbeat-53862091 (accessed November 2021). 
26. Hillier L (2021) Stats Roundup: the Impact of Covid-19 on Marketing \& Advertising. Econsultancy. https:// econsultancy.com/stats-roundup-coronavirus-impact-onmarketing-advertising/ (accessed January 2021).

27. UConn (2020) UConn Rudd Center for Food Policy and Obesity 'Food Advertising to Children and Teens Score' Reports. https://uconnruddcenter.org/research/foodmarketing/facts/ (accessed November 2021).

28. FACTS reports - UConn Rudd Center for Food Policy and Obesity. http://www.uconnruddcenter.org/facts-reports (accessed March 2020).

29. Python (2020) Difflib.py https://github.com/python/cpython/ blob/main/Lib/difflib.py (accessed December 2020).

30. Stream Hatchet (2019) Esports data and analytics - Stream Hatchet. Stream Hatchet. https://streamhatchet.com/ (accessed December 2020).

31. Team RC (2020) $R:$ A Language and Environment for Statistical Computing. Vienna, Austria: R Foundation for Statistical Computing.

32. Yau A, Adams J, Boyland EJ et al. (2021) Sociodemographic differences in self-reported exposure to high fat, salt and sugar food and drink advertising: a sectional analysis of 2019 UK panel data. BMJ Open 11, 1-12.

33. Pomeranz JL, Munsell CR \& Harris JL (2013) Energy drinks: an emerging public health hazard for youth. $J$ Public Health Policy 34, 254-271.

34. Seifert SM, Schaechter JL, Hershorin ER et al. (2011) Health effects of energy drinks on children, adolescents, and young adults. Pediatrics 127, 511-528.

35. Bailey RL, Saldanha LG, Gahche JJ et al. (2014) Estimating caffeine intake from energy drinks and dietary supplements in the US. Nutr Rev 72, 9-13.

36. Heckman MA, Sherry K \& de Mejia EG (2010) Energy drinks: an assessment of their market size, consumer demographics, ingredient profile, functionality, and regulations in the United States. Compr Rev Food Sci Food Saf 9, 303-317.

37. Larson N, Laska MN, Story M et al. (2015) Sports and energy drink consumption are linked to health-risk behaviours among young adults. Public Health Nutr 18, 2794-2803.

38. Reissig CJ, Strain EC \& Griffiths RR (2009) Caffeinated energy drinks - a growing problem. Drug Alcohol Depend 99, 1-10.

39. Tomkinson S \& Elliott J (2020) Hype source: G FUEL'S contemporary gamer persona and its navigation of prestige and diversity. Pers Stud 6, 22-37.

40. Emond JA, Fleming-Milici F, McCarthy J et al. (2020) Unhealthy Food Marketing on Commercial Educational Websites: remote Learning and Gaps in Regulation. Am J Prev Med 60, 1-5.
41. Khan I (2021) Twitch Is Having a Political Renaissance. Wired. https://www.wired.com/story/politicians-twitch-votersgeorgia-election/ (accessed October 2021).

42. Stephen B (2020) Twitch is going to Fund New Reality Programming. The Verge. https://www.theverge.com/2020/ 5/7/21250545/twitch-live-programming-reality-tv (accessed October 2021)

43. Kelly B, Halford JCG, Boyland EJ et al. (2010) Television food advertising to children: a global perspective. Am J Public Health 100, 1730-1736.

44. Ofcom (2019) Adults: Media Use and Attitudes Report 2019. Ofcom. https://www.ofcom.org.uk/research-and-data/medialiteracy-research/adults/adults-media-use-and-attitudes (accessed November 2021)

45. Twitch.tv (2021) Twitch Advertising. https://twitch advertising.tv/audience/ (accessed November 2021).

46. McGinnis JM, Gootman JA \& Kraak VI (2006) Committee on Food Marketing and the Diets of Children and Youth, Institute of Medicine. Food Marketing to Children and Youth: Threat or Opportunity? Washington, DC: National Academies Press.

47. Kunkel D, Wilcox B, Cantor J et al. (2004) Report of the APA Task Force on Advertising and Children. Washington, DC: American Psychological Association.

48. Boyland EJ, Nolan S, Kelly B et al. (2016) Advertising as a cue to consume: a systematic review and meta-analysis of the effects of acute exposure to unhealthy food and nonalcoholic beverage advertising on intake in children and adults. $\mathrm{Am} \mathrm{J}$ Clin Nutr 103, 519-533.

49. Boyland EJ \& Whalen R (2015) Food advertising to children and its effects on diet: review of recent prevalence and impact data. Pediatr Diabetes 16, 331-337.

50. Sadeghirad B, Duhaney T, Motaghipisheh S et al. (2016) Influence of unhealthy food and beverage marketing on children's dietary intake and preference: a systematic review and meta-analysis of randomized trials. Obes Rev 17, 945-959.

51. Anderson P, De Bruijn A, Angus K et al. (2009) Special issue: the message and the media: impact of alcohol advertising and media exposure on adolescent alcohol use: a systematic review of longitudinal studies. Alcohol Alcohol 44, 229-243.

52. Hingson RW, Heeren T \& Winter MR (2006) Age at drinking onset and alcohol dependence: age at onset, duration, and severity. Arch Pediatr Adolesc Med 160, 739-746.

53. Qutteina Y, De Backer C \& Smits T (2019) Media food marketing and eating outcomes among pre-adolescents and adolescents: a systematic review and meta-analysis. Obes Rev 20, 1708-1719. 